\title{
Entanglement-enhanced probing of a delicate material system
}

\author{
Florian Wolfgramm, ${ }^{1}$ Chiara Vitelli, ${ }^{2}$ Federica A. Beduini, ${ }^{1}$ Nicolas Godbout, ${ }^{3}$ and Morgan W. Mitchell ${ }^{1,4}$ \\ ${ }^{1}$ ICFO - Institut de Ciencies Fotoniques, Mediterranean Technology Park, 08860 Castelldefels (Barcelona), Spain \\ ${ }^{2}$ Center of Life Nanoscience at La Sapienza, Istituto Italiano di Tecnologia, Viale Regina Elena 255, 00181 Rome, Italy \\ ${ }^{3}$ COPL, Département de Génie Physique, École Polytechnique de Montréal, \\ C.P. 6079, Succ. Centre-ville, Montréal (Québec) H3C 3A7, Canada \\ ${ }^{4}$ ICREA - Institució Catalana de Recerca i Estudis Avançats, 08015 Barcelona, Spain
}

\begin{abstract}
Quantum metrology $y^{1}$ uses entanglement $t^{2 / 5}$ and other quantum effects ${ }^{6}$ to improve the sensitivity of demanding measurements ${ }^{7}$ ? . Probing of delicate systems demands high sensitivity from limited probe energy and motivates the field's key benchmark the standard quantum limit 10 . Here we report the first quantum-enhanced measurement of a delicate material system. We non-destructively probe an atomic spin ensemble by near-resonant Faraday rotation, a measurement that is limited by probeinduced scattering in quantum memory and spinsqueezing applications 6 (11)-13. We use narrowband, atom-resonant NOON states to beat the standard quantum limit of sensitivity by more than five standard deviations, both on a per-photon and a per-damage basis. This demonstrates quantum enhancement with fully realistic loss and noise, including variable-loss effects ${ }^{14-16}$. The experiment points the way to ultragentle probing of single atoms ${ }^{17}$, single molecules ${ }^{18}$, quantum gases ${ }^{19}$ and living cells ${ }^{20}$.
\end{abstract}

Linear interferometry with non-entangled states can reach at best the standard quantum limit (SQL) $\delta \phi=1 / \sqrt{N}$, where $\phi$ is the interferometric phase to be measured and $N$ is the number of probe particles. When increasing $N$ is not possible, quantum enhancement offers a practical advantage. A key example is interferometric gravitational-wave detection: in current operating conditions, squeezing improves sensitivity whereas increasing photon flux produces deleterious thermal effects ${ }^{9}$. Here we study an analogous number-limited scenario with very broad potential application: the probing of delicate systems, i.e., material systems which suffer significant damage due to the probing process. Examples are found in atomic ${ }^{17}$, molecular ${ }^{18}$, condensed matter ${ }^{19]}$ and biologica ${ }^{[20}$ science.

By the Kramers-Kronig relations, interferometric phase shifts are necessarily accompanied by absorption, implying deposition of energy in the probed medium. Absorption also degrades any quantum advantage, as described by recent theory $\sqrt{16 / 21 / 22}$. To further complicate matters, in real media the phase shift and absorption may depend on the same unknown quantity. To show advantage in a fully-realistic scenario, we probe a precisely understood material system using a quantum state permitting rigorous sensitivity and damage analysis.

Our delicate system is a ${ }^{85} \mathrm{Rb}$ atomic spin ensemble, similar to ensembles used for optical quantum memories ${ }^{12}$ and quantum-enhanced atom interferometry ${ }^{23}$. Non-destructive dispersive measurements on these systems, used for stor- age and readout of quantum information or to produce spin squeezing, are fundamentally limited by scattering-induced depolarization $6111 ; 13$. Both the measurement and damage properties of the ensemble can be calculated from first principles, making this an ideal model system.

We probe the ensemble with a polarization NOON state, a two-mode entangled state of the form $|N\rangle_{L}|0\rangle_{R}+$ $\exp [i \phi]|0\rangle_{L}|N\rangle_{R}$ (normalization omitted), in which $N$ particles are either all $L$ - or all $R$-circularly polarized. The use of entangled photons in the single-photon regime allows a rigorous quantification, by quantum state tomography and Fisher Information (FI) theory, of the information gained. To minimize losses while maximizing rotation, we employ a NOON state in a $7 \mathrm{MHz}$ spectral window detuned four Doppler widths from the nearest ${ }^{85} \mathrm{Rb}$ resonance. This requires matter-resonant indistinguishable photons, which we generate for the first time with an advanced downconversion source and ultra-narrow atom-based filter.

We study the ratio of $\mathrm{FI}$ to probe-induced damage, i.e., to photons absorbed (equivalently scattered) by the ensemble. Since both $\mathrm{FI}$ and damage scale linearly with the number of probe particles, the same advantage is gained for higher photon numbers, e.g., at the projection-noise level. We find the NOON state beats the SQL by $30 \pm 5 \%$ in information gained per photon and $23 \pm 4 \%$ per damage to the ensemble.

The setup is shown schematically in Fig. 1 b: narrowband NOON states at $\omega_{\mathrm{NOON}}$, the optical frequency of the $5^{2} \mathrm{~S}_{1 / 2} \mathrm{~F}=2 \rightarrow 5^{2} \mathrm{P}_{1 / 2} \mathrm{~F}^{\prime}=1$ transition of the $D_{1}$ line of ${ }^{87} \mathrm{Rb}$, are generated by cavity-enhanced spontaneous parametric down-conversion (CESPDC) and sent through the ensemble. The ensemble of ${ }^{85} \mathrm{Rb}$ atoms is contained in an anti-reflection coated vapor cell with internal length $L=75$ $\mathrm{mm}$, in a temperature-controlled oven at $70^{\circ} \mathrm{C}$, together with a $0.5 \%$ residual ${ }^{87} \mathrm{Rb}$ component. An applied axial magnetic field $B$ of up to $60 \mathrm{mT}$ produces resonantlyenhanced Faraday rotation of the optical polarization. After leaving the vapor cell, the photons are separated in polarization, frequency filtered, and detected with single-photon counters. Two counters on each polarization output record all possible outcomes, i.e., coincidences of $H H, H V$, and $V V$ polarizations are post-selected.

As seen in Fig. 2, all coincidence outcomes oscillate as a function of $B$, with two-fold super-resolution relative to the single-photon oscillation, visible in the singles counts due to a small imbalance between $H$ and $V$ in the input state. The interference visibilities are all $\geq 90 \%$, well above the $33 \%$ classical limit for $H H$ and $V V$ visibility ${ }^{24}$. Also shown are predicted coincidence rates $R_{i}=R_{0} P_{i}$, where $R_{0}$ is the 


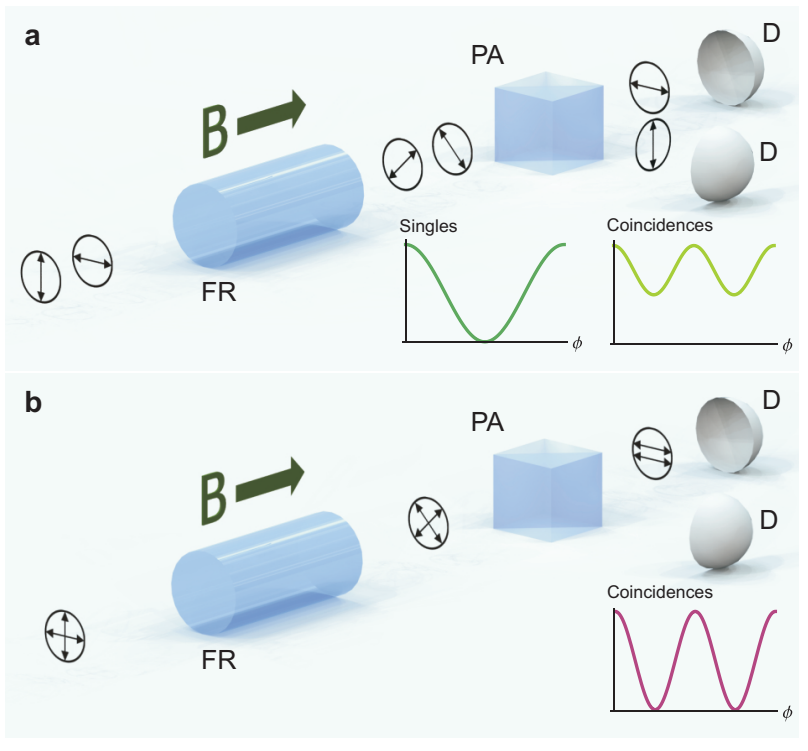

FIG. 1: NOON state probing of an atomic ensemble by Faraday rotation. Polarized photons pass through a rubidium vapor cell (FR) where they experience an optical rotation dependent on the applied magnetic field $\vec{B}$ (the Faraday effect) and are detected by a polarization analyzer (PA) with photon counting detectors (D). a With non-entangled photons, "singles detection" can give high visibility, while "coincidence detection" can give (low visibility) super-resolution. b By Hong-Ou-Mandel interference, NOON states can give both super-resolution and high visibility, providing more information per photon than possible without entanglement. In repeated probing, fewer photons are needed to achieve the same sensitivity, causing less damage to the sample.

input flux of pairs and

$$
P_{i}(B)=\operatorname{Tr}\left[\Pi_{i} T(B) \rho T^{\dagger}(B)\right],
$$

are the outcome probabilities. Here $\rho$ is the two-photon state before the cell, $B$ is the magnetic field, $T(B)$ describes the transmission through the cell, and $\Pi_{i}$ is the positive-operator-valued measure (POVM) element for the $i$ 'th outcome.

In the $\sigma_{ \pm}$basis, $T(B)=\operatorname{diag}\left(t_{+}^{2}, t_{+} t_{-}, t_{-} t_{+}, t_{-}^{2}\right)$, where $t_{ \pm}=\exp \left[i n_{ \pm} \omega_{\mathrm{NOON}} L / c\right]$ are transmission coefficients and $n_{ \pm}(B)$ are the complex refractive indices from a firstprinciples calculation (see supplementary information). In the $\mathrm{H}, \mathrm{V}$ basis, $\Pi_{H H}, \Pi_{H V}$ and $\Pi_{V V}$ are $\operatorname{diag}(1,0,0,0)$, $\operatorname{diag}(0,1,1,0)$, and $\operatorname{diag}(0,0,0,1)$, respectively. A completely analogous description is used for single photon probabilities. Due to atomic absorption and scattering, $\left|t_{ \pm}\right|<1$ so that $\sum_{i} P_{i}<1$ in general.

The $\rho$ in Eq. (1) is found by quantum state tomography 25 , i.e., a fit to the observed data using Eq. (1) and the known $T(B)$ and $\Pi_{i}$. We find a state, shown in Fig. 3 a, with high purity $\operatorname{Tr}\left[\rho^{2}\right]=0.88$, low photon distinguishability ${ }^{25}\left\langle\psi^{-}|\rho| \psi^{-}\right\rangle=0.02$, and high fidelity $\left\langle N_{\phi}|\rho| N_{\phi}\right\rangle=0.90$ with the NOON state $\left|N_{\phi}\right\rangle \equiv$ $\left(\left|2_{L} 0_{R}\right\rangle+\exp [2 i \phi]\left|0_{L} 2_{R}\right\rangle\right) / \sqrt{2}, \phi=0.22$. The phase $\phi$

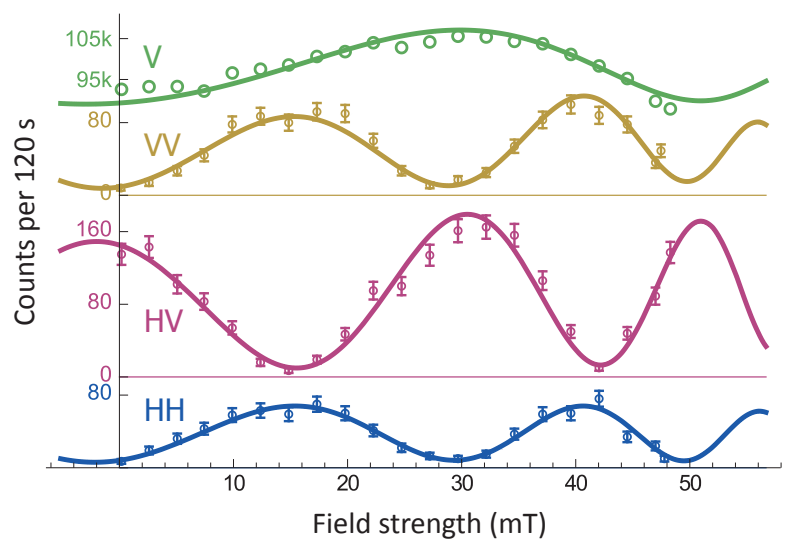

FIG. 2: High-visibility super-resolving Faraday rotation probing using optical NOON states. Top curve: for phase reference, singles detection rate ( $\mathrm{V}$ polarization) versus field strength $\mathrm{B}$ shows one oscillation in the range $\mathrm{B}=0$ to 50 mT. Other curves: coincidence detections $H H, H V$, and $V V$ show two oscillations in the same range (super-resolution) and high visibility. Symbols show measured data (no background subtracted), with $\pm 1 \sigma$ statistical uncertainties. Curves show model of Eq. 11 with state $\rho$ found by quantum state tomography.

is twice the input polarization rotation, which can be chosen to optimize sensitivity at any given value of $B$. Note that both rotation and absorption of the NOON photons is evident in Fig. 2, and contributes to the state reconstruction. A simultaneously-acquired time-correlation measurement, shown in Fig. 3 b confirms the spectral purity of the NOON state ${ }^{26}$. To our knowledge, this is the first demonstration of an atom-resonant state with multi-photon coherence.

We rigorously quantify the metrological advantage using the Fisher information (FI),

$$
\mathcal{I}(B) \equiv \sum_{i} P_{i}\left|\partial_{B} \ln P_{i}\right|^{2},
$$

where $P_{i}$ are the probabilities derived from the firstprinciples model in Eq. (1). The magnetic uncertainty
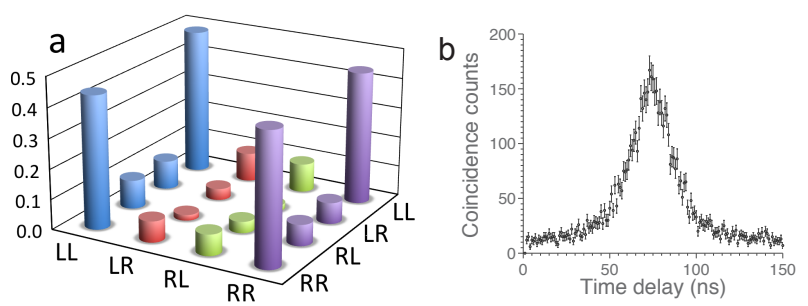

FIG. 3: NOON state characterization. a Density matrix $\rho$ (magnitudes only) from quantum state tomography, showing large coherence between $|L L\rangle$ and $|R R\rangle$ components. b Measured correlation of the filtered CESPDC pairs (no background subtracted). The absence of modulation at the $2 \mathrm{~ns}$ cavity round-trip time indicates the presence of a single cavity mode. 


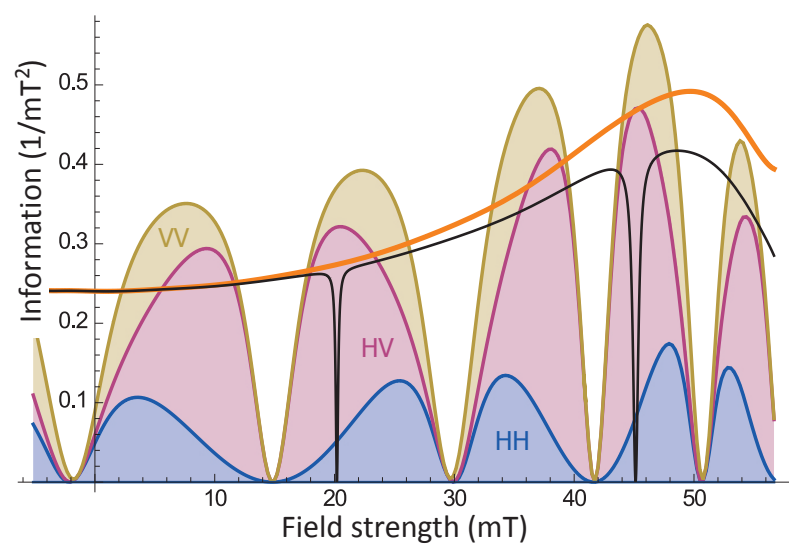

FIG. 4: Quantum-enhancement in probing of a delicate system, quantified by Fisher information (FI) per mean scattering (S) from the ${ }^{85} \mathrm{Rb}$ atomic ensemble. Thin black curve: FI/S for a single photon of an arbitrarily chosen linear input polarization. Thick orange curve: SQL, the largest FI/S obtainable with non-entangled states, found by numerical optimization of a single photon input and the measurement POVM. Shaded regions: contributions of $H H, H V$, and $V V$ outcomes to the FI/S with the experimental NOON state of Fig. 3. The NOON state surpasses the SQL by $40 \pm 6 \%$ (23 $\pm 4 \%$ with ${ }^{87} \mathrm{Rb}$ contaminant), giving a metrological advantage in the presence of fully realistic and parameterdependent losses. Low points in the FI are associated with extrema of the coincidence probability curves, i.e., regons of small signal sensitivity. For both single-photon and NOON state probing, the input polarization determines the position of the maxima, allowing high sensitivity measurement for any value of $B$.

is $\delta B \rightarrow(\mathcal{I} M)^{-1 / 2}$ in the practical scenario of $M \gg 1$ uses of the state 27 . On the basis of FI per input photon, the NOON state achieves an advantage $\mathcal{I}_{\text {NOON }} / 2 \mathcal{I}_{\mathrm{SQL}}=$ $1.30 \pm 0.05$ over the SQL found by numerical optimization over possible single-photon inputs and the POVM $\left\{\Pi_{i}\right\}$ (details in supplementary information). Despite the losses inherent in near-resonant probing of the ensemble, the NOON state offers a significant advantage.

The NOON state also gives an advantage in FI per scattered photon. This figure of merit quantifies the information gain per damage and differs from $\mathrm{FI}$ per input photon because the scattering is strongly B-dependent. We quantify scattering from the ${ }^{85} \mathrm{Rb}$ ensemble as $\mathcal{S}=\operatorname{Tr}\left[\rho \Pi_{\text {scat }}\right]$, where $\Pi_{\text {scat }} \equiv \operatorname{diag}\left(s_{++}, s_{+-}, s_{-+}, s_{--}\right)$in the $\sigma_{ \pm}$basis and the mean number of scattering events is $s_{a b} \equiv$ $2-\left|t_{a}^{(85)}\right|^{2}-\left|t_{b}^{(85)}\right|^{2}$, where $t_{ \pm}^{(85)}$ is the ${ }^{85} \mathrm{Rb}$ contribution to $t_{ \pm}$. A completely analogous calculation is made for singlephoton scattering. The NOON state gives an advantage $(\mathcal{I} / \mathcal{S})_{\mathrm{NOON}} /(\mathcal{I} / \mathcal{S})_{\mathrm{SQL}}=1.23 \pm 0.04$ over the SQL with our ensemble including the ${ }^{87} \mathrm{Rb}$ contaminant. If this were removed, the advantage would be $1.40 \pm 0.06$. Shown graphically in Fig. 4.

These post-selected results imply also an advantage without post-selection: with available detector efficiency 28 $\eta_{\text {det }}=0.95$ and source-to-detector path efficiency ${ }^{\sqrt{4}} \eta_{\text {path }}=$ 0.984 the quantum enhancement is $1.21 \pm 0.05$ per sent photon and $1.15 \pm 0.04$ per photon scattered from the ${ }^{85} \mathrm{Rb}$ ensemble $\left(1.31 \pm 0.06\right.$ without $\left.{ }^{87} \mathrm{Rb}\right)$. In the future, narrowband squeezed states 7 may allow larger enhancements ${ }^{4}$ with bright beams and higher detection efficiencies, although squeezing-induced damage is predicted in some scenarios 29 . See supplementary information. We note a previouslyunreported source of metrological advantage. In the ensemble, as in any material system, the loss depends on the measured quantity (here $B$ ). This dependence makes a positive contribution to the $\mathrm{FI}$ when it increases $\left|\partial_{B} \ln P_{i}\right|^{2}$, offsetting the well-known ${ }^{15} \mathrm{FI}$ reduction from $\sum_{i} P_{i}<1$.

We have demonstrated quantum-enhanced probing of an intrinsically delicate system, an atomic spin ensemble. Using narrowband, cavity-enhanced down-conversion, we generate a high-fidelity photonic NOON state tuned to an optical resonance of atomic rubidium. When used to probe a rubidium atomic ensemble, the NOON state surpasses the best possible non-entangled probe. The result shows that quantum entanglement can produce a more gentle measurement without sacrificing sensitivity. This is also to our knowledge the first use of multi-photon coherence in an atomic physics context. Quantum-enhanced probing of atomic ensembles paves the way for ultra-gentle probing of other delicate systems including exotic phases of cold quantum gases ${ }^{19}$, single molecules ${ }^{18}$ and living cells ${ }^{20}$.

\section{METHODS SUMMARY}

We use type-II CESPDC26/30/31 to produce $N=2$ NOON states. A $795 \mathrm{~nm}$ laser, stabilized to $\omega_{\mathrm{NOON}}$, the frequency of the $5^{2} \mathrm{~S}_{1 / 2} \mathrm{~F}=2 \rightarrow 5^{2} \mathrm{P}_{1 / 2} \mathrm{~F}^{\prime}=1$ transition of the $D_{1}$ line of ${ }^{87} \mathrm{Rb}$, is both used to stabilize the cavity length, and is frequency-doubled to pump the SPDC process. In the down-conversion process, the cavity resonantly enhances degenerate down-conversion into a $\mathrm{TEM}_{00}$ mode with bandwidth $7 \mathrm{MHz}$ around $\omega_{\text {NOON }}$. Non-degenerate emission, separated by at least the cavity's $490 \mathrm{MHz}$ free spectral range, is efficiently removed by filtering. The apparatus is described in detail in references 30 [31] By using a low pump power, we ensure that the emitted photons are in a two-photon state $\rho$, ideally the NOON state $|1\rangle_{H}|1\rangle_{V} \propto|2\rangle_{L}|0\rangle_{R}-|0\rangle_{L}|2\rangle_{R}$, with negligible fourphoton component. $H, V, L, R$ indicate horizontal, vertical, left circular (or $\sigma_{+}$) and right circular (or $\sigma_{-}$) polarizations, respectively.

The filter, described in references $32 \mid 33$, has an $80 \mathrm{MHz}$ FWHM passband centered on $\omega_{\text {NOON }}$ and $>35 \mathrm{~dB}$ outof-band rejection, so that only atom-tuned photons are detected. The single-mode character is evident in the double-exponential arrival-time distribution of the filtered NOON state, shown in Fig. 3.b. The combination of CESPDC and narrowband filtering produces a state with ideal characteristics for atomic probing: single-spatial mode, near-perfect indistinguishability, and extremely high temporal coherence. In a previous experiment 33 , we showed at least $94 \%$ of photon pairs are rubidium resonant. 
1 Giovannetti, V., Lloyd, S. \& Maccone, L. Advances in quantum metrology. Nature Photon. 5, 222-229 (2011).

2 Leibfried, D. et al. Toward Heisenberg-limited spectroscopy with multiparticle entangled states. Science 304, 1476-1478 (2004).

3 Mitchell, M. W., Lundeen, J. S. \& Steinberg, A. M. Superresolving phase measurements with a multiphoton entangled state. Nature 429, 161-164 (2004).

${ }^{4}$ Vahlbruch, H. et al. Observation of squeezed light with $10-$ db quantum-noise reduction. Phys. Rev. Lett. 100, 033602 (2008).

${ }^{5}$ Afek, I., Ambar, O. \& Silberberg, Y. High-NOON states by mixing quantum and classical light. Science 328, 879-881 (2010).

6 Napolitano, M. et al. Interaction-based quantum metrology showing scaling beyond the Heisenberg limit. Nature 471, 486-489 (2011).

7 Wolfgramm, F. et al. Squeezed-light optical magnetometry. Phys. Rev. Lett. 105, 053601 (2010).

${ }^{8}$ Shah, V., Vasilakis, G. \& Romalis, M. V. High bandwidth atomic magnetometery with continuous quantum nondemolition measurements. Phys. Rev. Lett. 104, 013601 (2010).

9 The LIGO Scientific Collaboration. A gravitational wave observatory operating beyond the quantum shot-noise limit. $\mathrm{Na}$ ture Phys. 7, 962-965 (2011).

10 Braunstein, S. L. Quantum metrology: Size isn't everything. Nature 440, 617-618 (2006).

11 Madsen, L. B. \& Mølmer, K. Spin squeezing and precision probing with light and samples of atoms in the gaussian description. Phys. Rev. A 70, 052324 (2004).

12 Julsgaard, B., Sherson, J., Cirac, J. I., Fiurasek, J. \& Polzik, E. S. Experimental demonstration of quantum memory for light. Nature 432, 482-486 (2004).

13 Koschorreck, M., Napolitano, M., Dubost, B. \& Mitchell, M. W. Quantum nondemolition measurement of large-spin ensembles by dynamical decoupling. Phys. Rev. Lett. 105, 093602 (2010)

14 Kacprowicz, M., Demkowicz-Dobrzanski, R., Wasilewski, W., Banaszek, K. \& Walmsley, I. A. Experimental quantumenhanced estimation of a lossy phase shift. Nature Photon. 4, 357-360 (2010).

15 Thomas-Peter, N. et al. Real-world quantum sensors: Evaluating resources for precision measurement. Phys. Rev. Lett. 107, 113603 (2011).

${ }^{16}$ Escher, B. M., de Matos Filho, R. L. \& Davidovich, L. General framework for estimating the ultimate precision limit in noisy quantum-enhanced metrology. Nature Phys. 7, 406-411 (2011).

17 Tey, M. K. et al. Strong interaction between light and a single trapped atom without the need for a cavity. Nature Phys. 4, 924-927 (2008).

18 Pototschnig, M. et al. Controlling the phase of a light beam with a single molecule. Phys. Rev. Lett. 107, 063001 (2011).

19 Eckert, K. et al. Quantum non-demolition detection of strongly correlated systems. Nature Phys. 4, 50-54 (2008).

${ }^{20}$ Carlton, P. M. et al. Fast live simultaneous multiwavelength four-dimensional optical microscopy. Proc. Natl. Acad. Sci. USA 107, 16016-16022 (2010).

21 Dorner, U. et al. Optimal quantum phase estimation. Phys. Rev. Lett. 102, 040403 (2009).

22 Banaszek, K., Demkowicz-Dobrzanski, R. \& Walmsley, I. A.
Quantum states made to measure. Nature Photon. 3, 673-676 (2009).

23 Schleier-Smith, M. H., Leroux, I. D. \& Vuletić, V. States of an ensemble of two-level atoms with reduced quantum uncertainty. Phys. Rev. Lett. 104, 073604 (2010).

${ }^{24}$ Afek, I., Ambar, O. \& Silberberg, Y. Classical bound for Mach-Zehnder superresolution. Phys. Rev. Lett. 104, 123602 (2010).

25 Adamson, R. B. A., Shalm, L. K., Mitchell, M. W. \& Steinberg, A. M. Multiparticle state tomography: Hidden differences. Phys. Rev. Lett. 98, 043601 (2007).

${ }^{26}$ Kuklewicz, C. E., Wong, F. N. C. \& Shapiro, J. H. Time-binmodulated biphotons from cavity-enhanced down-conversion. Phys. Rev. Lett. 97, 223601 (2006).

27 Fisher, R. A. Theory of statistical estimation. Proc. Camb. Phil. Soc. 22, 700-725 (1925).

${ }^{28}$ Lita, A. E., Miller, A. J. \& Nam, S. W. Counting near-infrared single-photons with 95\% efficiency. Opt. Express 16, 30323040 (2008).

${ }^{29}$ Auzinsh, M. et al. Can a quantum nondemolition measurement improve the sensitivity of an atomic magnetometer? Phys. Rev. Lett. 93, 173002 (2004).

30 Wolfgramm, F. et al. Bright filter-free source of indistinguishable photon pairs. Opt. Express 16, 18145-18151 (2008).

31 Wolfgramm, F., Cerè, A. \& Mitchell, M. W. NOON states from cavity-enhanced down-conversion: high quality and super-resolution. J. Opt. Soc. Am. B 27, A25-A29 (2010).

32 Cerè, A. et al. Narrowband tunable filter based on velocityselective optical pumping in an atomic vapor. Opt. Lett. 34, 1012-1014 (2009).

33 Wolfgramm, F., de Icaza Astiz, Y. A., Beduini, F. A., Cerè, A. \& Mitchell, M. W. Atom-resonant heralded single photons by interaction-free measurement. Phys. Rev. Lett. 106, 053602 (2011).

Acknowledgements: The authors thank A. Cerè, Y. A. de Icaza Astiz, M. Napolitano, N. Behbood, R. J. Sewell, and $M$. Hendrych for useful discussions. This work was supported by the Spanish Ministerio de Economia y Competitividad under the project Magnetometria Optica (No. FIS2011-23520), by the European Research Council starting grant "AQuMet", by Fundació Privada Cellex and by an Institute of Photonic Sciences - Ontario Centres of Excellence collaborative research programme. F.W. is supported by the Commission for Universities and Research of the Department of Innovation, Universities and Enterprises of the Catalan Government and the European Social Fund. N.G. acknowledges support from the National Sciences and Engineering Research Council of Canada.

Author contributions: F.W. and M.W.M. conceived and designed the project. F.W., C.V. and F.A.B. designed, constructed and tested the apparatus. F.W. acquired the data and performed the analysis. M.W.M. and N.G. performed the modelling and simulation. All authors contributed to the preparation of the manuscript. 


\section{SUPPLEMENTARY INFORMATION}

\section{A. Model of atomic vapor magneto-optical properties}

The cell, with an internal path of $75 \mathrm{~mm}$ and containing purified ${ }^{85} \mathrm{Rb}$ with a small $(0.5 \%)$ admixture of ${ }^{87} \mathrm{Rb}$, no buffer gas, and no wall coatings that might preserve polarization, is modeled as a thermal equilibrium, Doppler-broadened vapor subject to Zeeman shifts in the intermediate regime. The atomic structure is calculated by diagonalization of the atomic Hamiltonians $H_{\mathrm{At}}^{\text {(iso) }}=$ $H_{0}^{(\text {iso })}+H_{\mathrm{HFS}}^{(\text {iso }}+H_{\mathrm{Z}}^{(\text {iso })}$, where $H_{0}^{(\text {iso })}$ is the energy structure of the isotope ${ }^{\text {iso }} \mathrm{Rb}$ including fine-structure contribution, $H_{\mathrm{HFS}}^{(\text {iso) }}=g_{\mathrm{HFS}} \mathbf{J} \cdot \mathbf{I}$ is the hyperfine contribution, and $H_{\mathrm{Z}}^{(\text {iso })}=\mathbf{b} \cdot\left(g_{J} \mathbf{J}+g_{I} \mathbf{I}\right)$ is the Zeeman contribution. All atomic parameters are taken from references 12 . The matrices $H_{\mathrm{At}}^{(\mathrm{iso})}$ are numerically diagonalized to find field-dependent energy eigenstates, illustrated in Fig. 5. from which the complex linear optical polarizability is calculated, including radiative damping. The complex refractive index $n_{ \pm}$for $\sigma_{ \pm}$polarizations is computed including Doppler broadening and a temperature-dependent atom density given by the vapor pressure times the isotope fraction, and the transfer function for the cell is calculated from
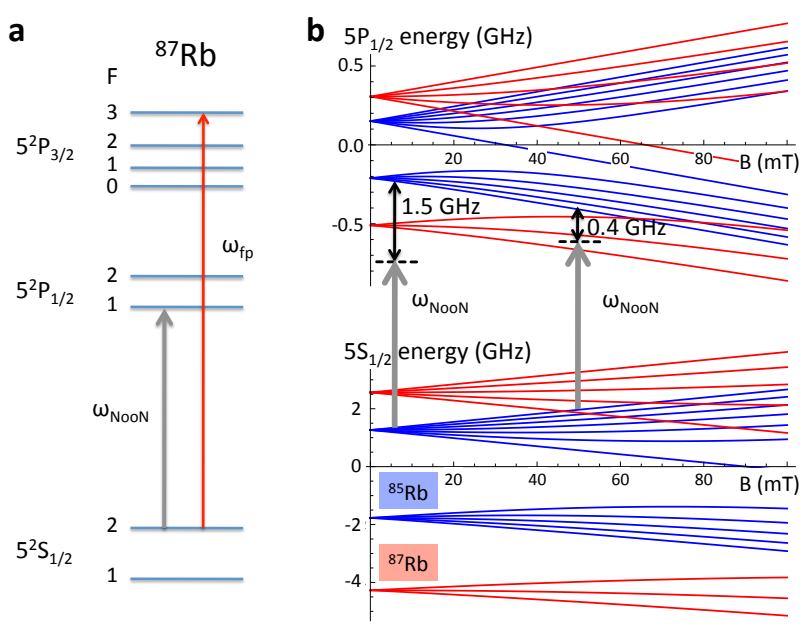

FIG. 5: Relevant energy level diagrams. a Energy levels of ${ }^{87} \mathrm{Rb}$ relevant to generation and filtering (not to scale). The frequency of the NooN state $\omega_{\text {NooN }}$ is tuned to the $5^{2} \mathrm{~S}_{1 / 2} \mathrm{~F}=2 \rightarrow 5^{2} \mathrm{P}_{1 / 2} \mathrm{~F}^{\prime}=1$ transition of the $D_{1}$ line of ${ }^{87} \mathrm{Rb}$. The optical pumping laser of the filter, with frequency $\omega_{\mathrm{fp}}$, addresses the $5^{2} \mathrm{~S}_{1 / 2} \mathrm{~F}=2 \rightarrow 5^{2} \mathrm{P}_{3 / 2} \mathrm{~F}^{\prime}=3$ transition of the $D_{2}$ line of ${ }^{87} \mathrm{Rb}$. The $15 \mathrm{~nm}$ separation from the detection wavelength allows a high extinction using interference filters centered on $\omega_{\text {NooN }}$. b $D_{1}$ energy levels of the probed ensemble versus field strength $B$, showing ${ }^{85} \mathrm{Rb}$ levels in blue and ${ }^{87} \mathrm{Rb}$ levels in red. At zero field $\omega_{\text {NOON }}$ is $1.5 \mathrm{GHz}$ detuned from the nearest ${ }^{85} \mathrm{Rb}$ transition. With increasing $B$, the nearest ${ }^{85} \mathrm{Rb}$ transition moves closer to resonance, increasing the Faraday rotation. The Doppler-broadened absorption begins to overlap $\omega_{\text {NOON }}$ near $B=50 \mathrm{mT}$.

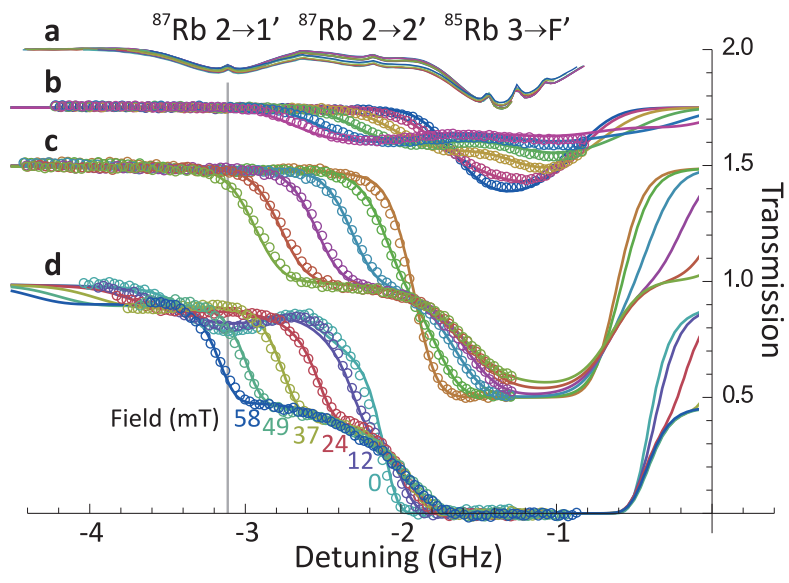

FIG. 6: Spectroscopic characterization of the rubidium atomic ensemble. Circles show measured values, curves show predictions of first-principles model (see text). a Saturatedabsorption spectra acquired with a natural-abundance cell at room temperature, as a frequency reference. Horizontal axis shows detuning from the center of the $D_{1}$ spectral line. b-d Transmission spectra for the cell containing ${ }^{85} \mathrm{Rb}$ plus $0.5 \%$ ${ }^{87} \mathrm{Rb}$ at temperatures of $22^{\circ} \mathrm{C}, 53^{\circ} \mathrm{C}$ and $83^{\circ} \mathrm{C}$, respectively. For each temperature, spectra with measured field strengths (in $\mathrm{mT}$ ) of $0,12,24,37,49$, and 58 are shown, in order of increasing line broadening. Grey vertical line shows $\omega_{\text {NOON }}$, the probe detuning. This operating point gives strong Faraday rotation with low absorption over the range 0-49 $\mathrm{mT}$. Absorption from the small residual ${ }^{87} \mathrm{Rb}$ component can be seen in d. For clarity, parts a-c have been offset vertically by $1,0.75$, and 0.5 , respectively.

the integral of the index along the beam path, including the measured drop in field strength of $15 \%$ from the center to the faces of the cell.

Transmission spectra, acquired with a low-power laser passing through the probe beam path, are used to validate the model, as shown in Fig. 6 The frequency scale of the spectra is determined from simultaneous saturated absorption spectroscopy. Spectra taken at temperatures $22^{\circ} \mathrm{C}$, $53^{\circ} \mathrm{C}$, and $83^{\circ} \mathrm{C}$, and fields in the range $0-58 \mathrm{mT}$ are used to determine the isotope fraction and to calibrate the field and temperature indicators, i.e., the current in the coils and the resistance of a thermistor near the cell, respectively. The transfer functions are shown in Fig. 7

\section{B. Fisher information}

The sensitivity of the measurement is computed using the Fisher Information (FI) ${ }^{3}$

$$
\mathcal{I}(B)=\sum_{i} P_{i}\left(\partial_{B} \ln P_{i}\right)^{2} .
$$

Fig. 8 shows $\mathcal{I} / 2$, the $\mathrm{FI}$ per photon for the experimental NOON state $\rho$ shown in Fig. 3 In an experiment with $N_{\text {tot }} \gg 1$ total photons, the resulting magnetic uncertainty 

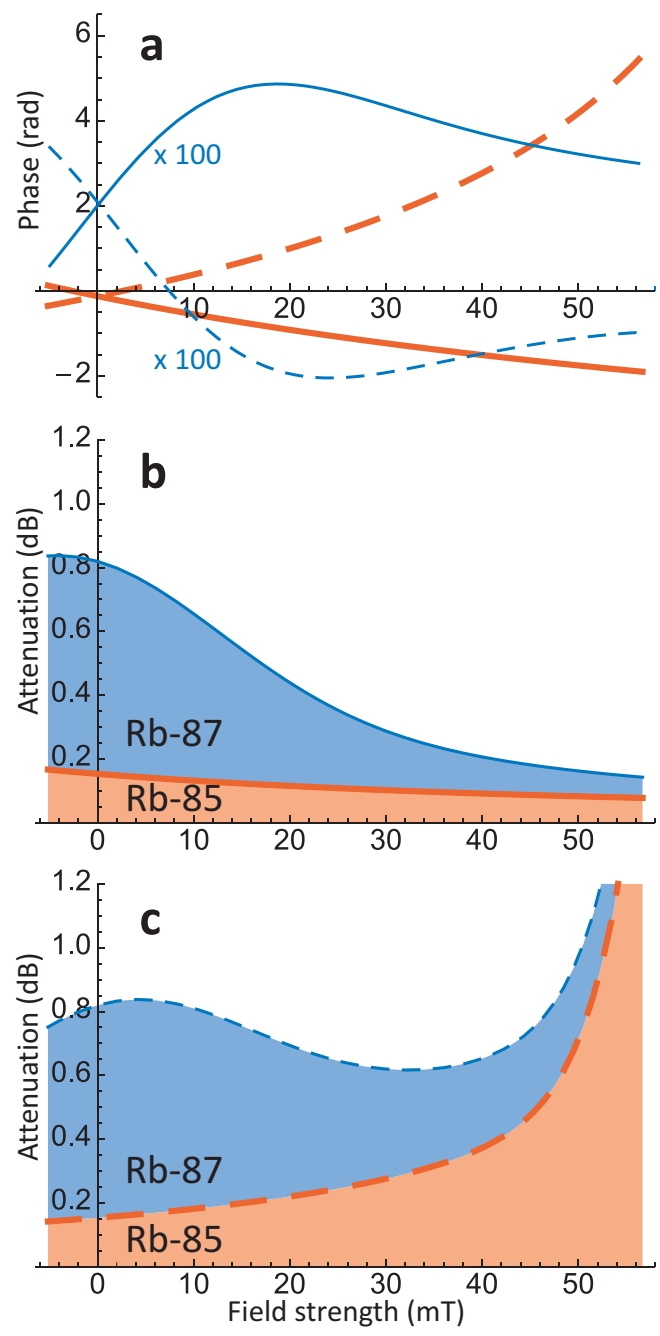

FIG. 7: Magneto-optical properties of the atomic ensemble, for probe light at $\omega_{\text {NOON }}$, computed from model. Polarizations are shown by solid $\left(\sigma_{+}\right)$and dashed $\left(\sigma_{-}\right)$curves. Isotopic contributions are shown by thick orange $\left({ }^{85} \mathrm{Rb}\right)$ and thin blue $\left({ }^{87} \mathrm{Rb}\right)$ lines. a Phase retardation vs. field strength. The differential $\sigma_{ \pm}$retardation produces optical rotation. Contribution of ${ }^{85} \mathrm{Rb}$ is two orders of magnitude larger than that of ${ }^{87} \mathrm{Rb}$, as expected from the isotopic ratio, and in the opposite sense. b, c Attenuation vs. field strength for $\sigma_{+}, \sigma_{-}$polarizations.

is $\delta B=\left(N_{\mathrm{tot}} \mathcal{I} / 2\right)^{-1 / 2}$. Also shown is the standard quantum limit (SQL) for this figure of merit, i.e., the best possible $\mathrm{FI}$ per photon with non-entangled states, found by numerical optimization over possible single-photon inputs and the POVM $\left\{\Pi_{i}\right\}$. The NOON state achieves $1.30 \pm 0.05$ improvement over the SQL, at a field $B=37 \mathrm{mT}$. All uncertainties given are one sigma statistical error. As with non-entangled photons (shown also in Fig. 8), the point of maximum sensitivity can be selected by varying the input polarization or by applying a bias magnetic field.

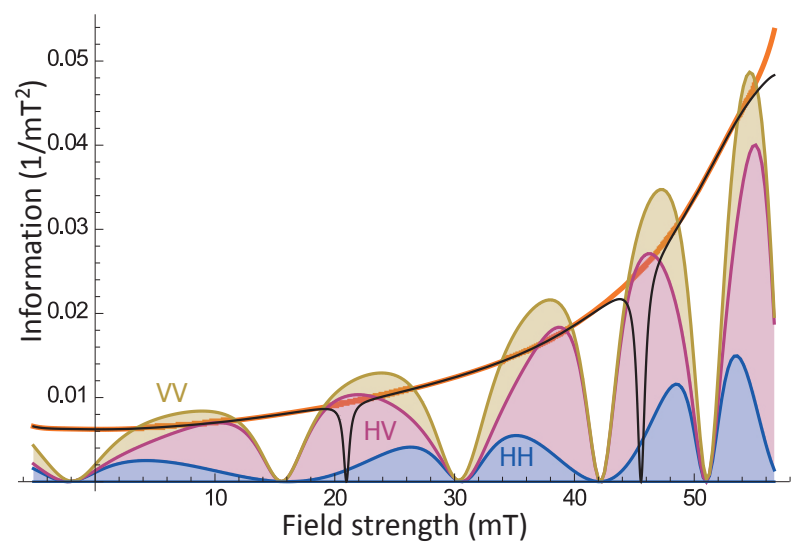

FIG. 8: Fisher information (FI) per input photon in Faraday rotation probing of the ensemble. Thin black curve shows FI for non-entangled photons of an arbitrarily chosen linear input polarization. Thick orange curve shows the "standard quantum limit," the largest FI obtainable with non-entangled states. Solid regions indicate contributions of $H H, H V$, and $V V$ outcomes to the NOON state FI, calculated using $\rho$ from Fig. 3 In all cases FI grows with increasing field, due to increased proximity to the ${ }^{85} \mathrm{Rb}$ resonances.

\section{Fisher information per damage}

The NOON state also gives an advantage in FI per scattered photon, the figure of merit for low-damage probing. We quantify scattering from the ${ }^{85} \mathrm{Rb}$ ensemble as $\mathcal{S}=\operatorname{Tr}\left[\rho \Pi_{\text {scat }}\right]$, where $\Pi_{\text {scat }} \equiv \operatorname{diag}\left(s_{++}, s_{+-}, s_{-+}, s_{--}\right)$ in the $\sigma_{ \pm}$basis and the mean number of scattering events is $s_{a b} \equiv 2-\left|t_{a}^{(85)}\right|^{2}-\left|t_{b}^{(85)}\right|^{2}$, where $t_{ \pm}^{(85)}$ is the ${ }^{85} \mathrm{Rb}$ contribution to $t_{ \pm}$. A completely analogous calculation is made for single-photon scattering. As above, the the SQL for this figure of merit is found by numerical optimization. As shown in Fig. 4 the NOON state gives an advantage $(\mathcal{I} / \mathcal{S})_{\mathrm{NOON}} /(\mathcal{I} / \mathcal{S})_{\mathrm{SQL}}=1.40 \pm 0.06$ over the SQL.

\section{State reconstruction and statistical error of Fisher information}

The state $\rho$ is found by quantum state tomography from the data shown in Fig. 2, that is, by numerical search for the $\rho$ that minimises the chi-squared distance between the predictions of Eq. 1 and the observed data. The optimized state is reported as the reconstructed $\rho$. To find statistical errors for the Fisher information, we search in the vicinity of $\rho$ for states $\rho^{\prime}$ which maximize or minimize the Fisher information with a chi-squared distance at most $\delta$ larger than the optimum chi-squared. This establishes a statistical error which accounts for the non-linearity of the reconstruction procedure. The error ranges reported throughout are for $\delta=1$, corresponding to $\pm 1 \sigma$ in the case of a simple linear reconstruction. 


\section{E. Relation to state-of-the-art detection}

Thomas-Peter et al! ${ }^{4}$ consider the effect of detector inefficiency and derive visibility thresholds for metrological advantage in scenarios with constant loss. These thresholds are not directly applicable to our scenario with polarizationdependent, parameter-dependent losses. We note that intrinsic losses are included already in the FI calculation, and because they are field-dependent, provide some information about $B$, offsetting the loss of $\mathrm{FI}$ due to non-arrival of pairs.
Constant extrinsic losses including detector inefficiency reduce the NOON FI by $\eta_{\mathrm{ex}}^{2}$ versus $\eta_{\mathrm{ex}}$ for any single-photon state. Current technology can achieve extrinsic efficiency of $\eta_{\text {ex }}=\eta_{\text {det }} \eta_{\text {path }}$, with detector efficiency ${ }^{5} \eta_{\text {det }}=0.95$ and source-to-detector path efficiency ${ }^{6}$ (including escape from the source cavity) $\eta_{\text {path }}=0.984$. With current technology, the NOON state demonstrated here gives a quantum enhancement of $1.21 \pm 0.05$ per sent photon and $1.15 \pm 0.04$ per photon scattered from the ${ }^{85} \mathrm{Rb}$ ensemble $(1.31 \pm 0.06$ if the ${ }^{87} \mathrm{Rb}$ contaminant is removed).
${ }^{1}$ Steck, D. A. Rubidium 85 D line data. Available online at http://steck.us/alkalidata (Revision 2.1.5, 19 September 2012).

2 Steck, D. A. Rubidium 87 D line data. Available online at http://steck.us/alkalidata (Revision 2.1.4, 23 December 2010).

3 Braunstein, S. L. \& Caves, C. M. Statistical distance and the geometry of quantum states. Phys. Rev. Lett. 72, 3439-3443 (1994)

4 Thomas-Peter, N. et al. Real-world quantum sensors: Eval- uating resources for precision measurement. Phys. Rev. Lett. 107, 113603 (2011).

${ }^{5}$ Lita, A. E., Miller, A. J. \& Nam, S. W. Counting near-infrared single-photons with $95 \%$ efficiency. Opt. Express 16, 30323040 (2008).

${ }^{6}$ Vahlbruch, H. et al. Observation of squeezed light with 10 dB quantum-noise reduction. Phys. Rev. Lett. 100, 033602 (2008). 